\title{
THE WAVE MAP PROBLEM
}

Manoussos G. Grillakis

Dept. of Mathematics

University of Maryland

College Park MD 20742

\section{Introduction :}

The simplest way to introduce the problem is by considering an unknown map,

$$
\mathbf{u}=\left(u^{1}, u^{2}, \ldots u^{n}\right):\left\{R^{n+1} ; \eta_{\mu \nu}\right\} \mapsto\left\{M^{n} ; g_{j k}\right\}
$$

between the Minkowski space $R^{n+1}$ equiped with the metric

$$
\eta_{\mu \nu}:=\operatorname{diag}\{-1,1,1 \ldots, 1\} \quad, \quad \mu \nu=\{0,1, \ldots n\}
$$

and some n-dimensional Riemannian manifold $M$ with metric $g_{j k}$. The map should be the critical point of the following Lagrangian,

$$
L(u):=\int_{R^{n+1}}\left\{g_{j k}(\mathbf{u}) \eta^{\mu \nu} \nabla_{\mu} u^{j} \nabla_{\nu} u^{k}\right\} d \mathbf{x} .
$$

Computing the variation of this Lagrangian gives the system of equations

$$
\square u^{j}+\Gamma_{k l}^{j}(\mathbf{u})\left\{\nabla_{\mu} u^{k} \nabla^{\mu} u^{l}\right\}=0,
$$

where $\square:=-\nabla_{\mu} \nabla^{\mu}$ is the D'Alembertian operator and $\Gamma_{k l}^{j}$ are the Christoffel symbols on the manifold $M$. In the formulation of the problem and in what follows below I will use the summation convention namely summation over repeated indices is imlplied and I raise indices by the formula

$$
\nabla^{\mu}:=\eta^{\mu \nu} \nabla_{\nu}
$$

In order to be able to solve (0.4), one has to prescribe intial data on some space-like hypersurface. For example, denoting the first coordinate $x^{0}:=t$, we can prescribe at $t=0$ the map $u^{j}\left(0, x^{1}, \ldots, x^{n}\right)$ and its time derivatives $u_{, t}\left(0, x^{1}, \ldots, x^{n}\right)$. Equations (0.4) together with the initial data form the wave map problem. See [12] for a similar but harder problem.

Standard arguments can show that if the initial data are sufficiently smooth, then a smooth solution of (0.4) exists for sufficiently small times. The natural 
question one can ask is, whether given smooth data the solution of (0.4) exists for all times and if this is not the case to describe the possible loss of smoothness of the map $\mathbf{u}$. In general one expects that the behaviour of the solution is determined by an interplay between the dimension $n$ of the underlying Minkowski space and the geometric properties of the target manifold $M$. This idea is justified to some extend but recent investigations, [2], indicate that if $n$ is large, for example $n \geq 7$, then the geometry of the target manifold is not important and solutions of (0.4) do blow-up in finite time. The most interesting and rich cases are $n=2,3$. In general one expects that if the target manifold is negatively curved then solutions of $(0.4)$ are regular, while for positively curved manifold solutions explode in finite time. This is justified to some degree, see $[1,5]$.

There is a distinguished quantity that is crucial for this problem. The best way to introduce it, is through the Energy-Momentum-tensor, namely define the tensor

$$
T_{\mu \nu}:=g_{j k} u_{, \mu}^{j} u_{, \nu}^{k}-\eta_{\mu \nu}\left\{\eta^{\alpha \beta} g_{j k} u_{, \alpha}^{j} u_{, \beta}^{k}\right\}
$$

then $T_{\mu \nu}$ satisfies,

$$
\nabla_{\mu} T_{\nu}^{\mu}=0 \quad \nu=(0,1 \ldots n)
$$

Now look at the quantity $T_{00}$. It is a positive quantity and is preserved by the flow i.e. $\int_{R^{n}} T_{00} d \mathbf{x}=$ const.. This is the total energy of the system. One would like to use this as a first step in order to obtain global solutions. Assume that we can show that a local solution exists for rough intial data that have only finite energy. Then the conservation of energy automatically gives a global solution. This idea can only work for $n=2$ which is the critical dimension of this problem, see [3].

If we assume that $n=2$ the problem is critical and there is hope that refinements of existing methods can give definite answers to the problem. Let me rephrase the equations using the language of complex variables. We are seeking a map

$$
z(\mathbf{x}):\left\{R^{2+1} ; \eta_{\mu \nu}\right\} \mapsto\{D ; g(z, \bar{z}) d z d \bar{z}\},
$$

from $R^{2+1}$, Minkowski space into a subset $D$ of the complex plane $C$ with metric given by the conformal factor $g(z, \bar{z})$. This map is the critical point of the Lagrangian

$$
L(z, \bar{z}):=\int_{R^{2+1}}\left\{g(z, \bar{z}) \nabla_{\mu} z \nabla^{\mu} \bar{z}\right\} d \mathbf{x}
$$

and the equation to be solved is

$$
\square z-\left(\log (g(z, \bar{z}))_{, z}\left\{\nabla_{\mu} z \nabla^{\mu} z\right\}=0 .\right.
$$

There are certain natural conjectures for this problem .

Conjecture 1 : Assume that $g=4 /\left(1+|z|^{2}\right)^{2}$, i.e. the target manifold is the unit sphere $S^{2}$. For some smooth initial data the solution of $(0.10)$ explodes in finite time in some manner to be described. 
Conjecture 2 : Assume that $g=4 /\left(1-|z|^{2}\right)^{2}$, i.e. $D:=\{|z|<1\}$ and the target manifold is the hyperbolic plane $H^{2}$. The map remains regular provided that the initial data are smooth.

Conjecture 3 : Assume that $n=3$ and that $M:=H^{3}$ i.e. the hyperbolic three dimensional manifold. The solution of $(0.4)$ is smooth provided that the initial data are smooth.

Conjecture 3 is hopeless at present. However if $M:=S^{3}$ solutions do blow-up in finite time, see [5].

Assuming some symmetry simplifies the problem and certain things are known in these special cases. Assume that $z(t, r)$ where $r:=|\mathbf{x}|$ and that the target manifold is $H^{2}$ then the solutions of $(0.10)$ are regular provided that the initial data are smooth. This is a remarkable result, proved in [1]. The ouline of the method is illuminating and proceeds as follows. First the authors show that the energy does not "concentrate" inside a characteristic cone. Then they use the fact that the solution can loose regularity only at the central time-line, $r=0$, in order to estimate derivatives of the map in a weighted $L^{\infty}$ norm.

There is another symmetry assumption that reduces the problem to a manageable one. Choose coordinates $(t, \mathbf{x})=\left(t, r e^{i \theta}\right)$ and $z=R e^{i \phi}$. Next make the identification $\phi:=k \theta$ where $k=$ integer. This simplification removes the derivatives from the the nonlinear term in (0.10) and the energy controls the range of the map, see [5], [6], [11].

If one wishes to avoid the complications arising from the geometry of the target manifold then the following conjecture must be true.

Conjecture 4: Assume that the initial data are smooth and the initial energy is sufficiently small. Then solutions of (0.10) remain regular for all time.

The above conjecture states that small energy implies regularity. An even simpler conjecture is the following.

Conjecture 5 : Assume that there exist a smooth solution of $(0.10)$ in the domain $R^{2} \times[0, T)$ (this is guaranteed by the local existence theorem, which is standard). Now asume that $z \in C\left(R^{2} \times[0, T]\right)$. Show that $z$ is in fact smooth in $R^{2} \times[0, T]$. Hence again by the local existence theorem the solution is smooth in $R^{2} \times\left[0, T_{1}\right)$ for some $T_{1}>T$.

The conjecture above states that continuity of the map implies regularity and gives some information about the possible way that a solution might loose smoothness.

For the reason that I explained above there is a considerable amount of work done on proving local existence of solutions with as rough initial data as possible. The best result in this direction is [7]. One can outline this method as follows, observe first that,

$$
\square\left\{\frac{z^{2}}{2}\right\}=z \square z-\left\{\nabla_{\mu} z \nabla^{\mu} z\right\} .
$$

Then equation $(0.10)$ can be rewritten as

$$
\square z+H(z, \bar{z}) \bar{z} \square z^{2}=0 \quad,
$$


where $H$ is some function. The construction of the solution is accomplished in the following norm. Consider a function $f(t, x)$ whose Fourier transform I will denote by $\hat{f}(\tau, \xi)$ Now define the norm

$$
N_{\delta, s}(f):=\left\|(1+|| \tau|-| \xi||)^{\delta}(1+|\tau|+|\xi|)^{s} \hat{f}(\tau, \xi)\right\|_{L^{2}\left(R^{2+1}\right)} .
$$

This type of norm was introduced in [8], see also [9]. The key ingredient in the construction are two inequalities. For $\epsilon>0$ arbitrarily small we have

$$
\begin{aligned}
N_{\delta, s}\left(f^{2}\right) \leq C N_{\delta, s}^{2}(f) \quad & ; \quad N_{\delta, 0}(f g) \leq C N_{\delta, 0}(f) N_{\delta, s}(g) \\
\delta=\frac{1}{2}-\epsilon \quad & , \quad s=1+2 \epsilon
\end{aligned}
$$

See [3], [4]. These inequalities are not optimal, one of the norms on the right hand side should be replaced by $L^{\infty}$.

\section{References :}

1. Christodoulou, D. Tahvildar-Zadeh, A. On the regularity of spherically symmetric wave maps. CPAM XLVI (1993), 1041-1091.

2. Cazenave, T. Shatah, J. Tahvildar-Zadeh, A. Harmonic Maps of the Hyperbolic Space and Development of Singularities in Wave Maps and Yang-Mills Fields. (Preprint) (1997).

3. Grillakis, G.M. Apriori Estimates and Regularity of Nonlinear Waves. Proceedings of ICM Zürich, Switzerland 1995 (1187-1194).

4. Klainerman, S. Machedon, M. On the regularity properties of a model problem related to wave maps. Duke Math. Journ. 87 no. 3 553-589, (1997).

5. Shatah, J. Tahvildar-Zadeh, A. On the Cauchy problem for equivariant wave maps. CPAM 47 pp. 719-754, (1993).

6. Shatah, J. Tahvildar-Zadeh, A. Regularity of harmonic maps from the Minkowski space into rotationally symmetric manifolds. CPAM 45 (1992), 947971.

7. Tataru, D. Local and Global solutions for Wave Maps. Preprint.

8. Beals, M. Self-spreading and strength of singularities for solutions to semilinear wave equations. Ann. of Math. 1181983 187-214.

9. Bourgain J. Fourier transform restiction phenomena for certain lattice subsets and applications to nonlinear evolution equations. GAFA 3 (1993), 209-262.

10. Grillakis, M.G. Energy Estimates and the Wave Map Problem. To appear in Comm. in PDE.

11. Grillakis, M.G. Classical solutions for the equivariant wave map. (preprint).

12. Weinstein, G. On Rotating Black Holes in Equilibrium in General Relativity. CPAM $43,903-948$. 